\title{
Importance of luminal membrane mesothelin expression in intraductal papillary mucinous neoplasms
}

\author{
TAKAHIRO EINAMA ${ }^{1,2,3}$, HIROFUMI KAMACHI ${ }^{1}$, HIROSHI NISHIHARA ${ }^{4}$, SHIGENORI HOMMA ${ }^{1}$, \\ HIROMI KANNO $^{5}$, MARIN ISHIKAWA ${ }^{5}$, FUTOSHI KAWAMATA ${ }^{1}$, YUJI KONISHI ${ }^{1}$, MASANORI SATO ${ }^{1}$, \\ MUNENORI TAHARA $^{1}$, KUNIAKI OKADA ${ }^{6}$, SHUNJI MURAOKA $^{7}$, TOSHIYA KAMIYAMA $^{1}$, \\ AKINOBU TAKETOMI $^{1}$, YOSHIHIRO MATSUNO ${ }^{8}$, HIROYUKI FURUKAWA $^{3}$ and SATORU TODO $^{1}$ \\ ${ }^{1}$ Department of General Surgery, Hokkaido University Graduate School of Medicine, Sapporo, Hokkaido 060-8638; \\ ${ }^{2}$ Department of Surgery, Hokkaido Social Work Association Obihiro Hospital, Obihiro, Hokkaido 080-0805; \\ ${ }^{3}$ Division of Gastroenterological and General Surgery, Asahikawa Medical University, Asahikawa, Hokkaido 078-8510; \\ ${ }^{4}$ Department of Translational Pathology, Hokkaido University Graduate School of Medicine; ${ }^{5}$ Department of Pathology, \\ Laboratory of Cancer Research, Hokkaido University School of Medicine, Sapporo, Hokkaido 060-8638; \\ Departments of ${ }^{6}$ Surgery and ${ }^{7}$ Pathology, JA Sapporo Kosei Hospital, Sapporo, Hokkaido 060-0033; \\ ${ }^{8}$ Department of Surgical Pathology, Hokkaido University Hospital, Sapporo, Hokkaido 060-8638, Japan
}

Received February 11, 2014; Accepted November 28, 2014

DOI: $10.3892 / 01.2015 .2969$

\begin{abstract}
The present study demonstrated that luminal membrane mesothelin expression is a reliable prognostic factor in gastric cancer. Intraductal papillary mucinous neoplasms (IPMNs) often exhibit a spectrum of dysplasia, ranging between adenoma and carcinoma. Therefore, an immunohistochemical analysis of mesothelin expression in IPMN was performed in the present study, focusing on the localization of mesothelin. IPMNs were classified into two groups, IPMNs associated with invasive carcinoma and low-high (L-H) grade dysplasias. The tumors were classified as mesothelin-positive or -negative and in the mesothelin-positive cases, the localization of mesothelin was evaluated as luminal membrane- or cytoplasmic-positive. Among the 37 IPMNs, mesothelin expression was observed in 21 samples (56.8\%), including 46.2\% (12 out of 26) of the $\mathrm{L}-\mathrm{H}$ dysplasia and $81.8 \%$ (9 out of 11) of the invasive carcinoma samples $(\mathrm{P}=0.071)$. Luminal membrane localization was observed in 10 samples $(27 \%)$, including $15.4 \%(4 / 26)$ of the L-H dysplasia samples and $54.5 \%$ (6 out of 11 ) of the invasive carcinoma samples $(\mathrm{P}=0.022)$. Six patients experienced post-operative recurrence, with five of the recurrent tumors exhibiting mesothelin expression and all six exhibiting luminal membrane localization. It was concluded that immunohistochemical examinations for mesothelin expression and
\end{abstract}

Correspondence to: Dr Takahiro Einama, Department of General Surgery, Hokkaido University Graduate School of Medicine, Kita-Ku, Kita 14, Nishi 7, Sapporo, Hokkaido 060-8638, Japan E-mail: titiuehahaue@hotmail.com

Key words: mesothelin, intraductal papillary mucinous neoplasms, luminal membrane expression localization are clinically useful for prognostic assessments and decision making regarding further treatment subsequent to surgical procedures in patients with IPMN.

\section{Introduction}

Mesothelin is a $40-\mathrm{kDa}$ cell-surface glycoprotein that is expressed in the normal mesothelial cells lining the pleura, pericardium and peritoneum (1-2). Overexpression of mesothelin has also been identified in several types of cancer, including mesothelioma, ovarian cancer and pancreatic cancer (3-6). The full-length human mesothelin gene codes for a 71-kDa precursor protein, which is cleaved by furin-like proteases into a $40-\mathrm{kDa} \mathrm{C}$-terminal fragment that remains membrane bound and a $31-\mathrm{kDa} \mathrm{N}$-terminal fragment, which is secreted into the blood. The C-terminal $40-\mathrm{kDa}$ fragment is termed mesothelin and is attached to the cell membrane via a glycosyl-phosphatidylinositol (GPI) anchor (1).

The biological function of mesothelin is not fully understood, although previous studies have suggested that mesothelin overexpression increases cell proliferation and migration (7). In pancreatic cancer, a previous study found that the expression of mesothelin is associated with unfavorable outcomes (8). Furthermore, the localization of mesothelin in gastric cancer, extrahepatic bile duct cancer and colorectal adenocarcinoma was also investigated in each study. It was found that the expression of mesothelin at the luminal membrane was a reliable prognostic factor, suggesting that membrane-localized mesothelin plays a functionally significant role in promoting aggressive behavior in the aforementioned cancers (9-11).

Intraductal papillary mucinous neoplasm (IPMN) often exhibits a spectrum of dysplasias, ranging between adenoma and carcinoma in the same lesion (12). To date, however, there have not been any studies regarding the significance of mesothelin expression in IPMN. Therefore, an immunohistochemical 
analysis of mesothelin expression in IPMN was performed in the present study, focusing on the localization of mesothelin, determining whether mesothelin is present in the luminal membrane or cytoplasm.

\section{Materials and methods}

Patients and tumor specimens. The present study was performed with the approval of the Internal Review Board on Ethical Issues of Hokkaido University Hospital (Sapporo, Hokkaido, Japan), and written informed consent was obtained from the patients. The subjects consisted of 37 patients who underwent surgery with curative intent for IPMN between January 2000 and December 2006 at the Department of General Surgery (Hokkaido University, Graduate School of Medicine, Sapporo, Japan) or JA Sapporo Kosei Hospital (Sapporo, Japan). The IPMNs were classified into two groups, IPMNs associated with invasive carcinoma, termed invasive carcinomas, and those associated with low to high $(\mathrm{L}-\mathrm{H})$ grade dysplasias, termed L-H dysplasias, according to the 2010 World Health Organization criteria (12). The clinicopathological characteristics of these cases are summarized in Table I.

Out of the 37 patients with IPMN, 26 (70.3\%) were classified as possessing L-H grade dysplasia and the remaining 11 patients $(39.7 \%)$ were categorized as possessing invasive carcinoma. The mean age of the cohort was 67.2 years (standard deviation, \pm 9.7 years). In total, 24 patients (64.9\%) were male and the remaining 13 patients $(35.1 \%)$ were female. The tumors were classified as branch duct type tumors in 25 cases $(67.6 \%)$, main duct tumors in 10 cases $(27.0 \%)$, and combined type tumors in two cases $(5.4 \%)$. Mural nodules were identified in 31 patients $(83.8 \%)$. Of the 37 patients, four succumbed to the disease, and the median follow-up period of the surviving 33 patients was 50.4 months (range, 5.9-103.0 months).

Formalin-fixed paraffin-embedded tissue blocks were prepared from the tumor specimens. The sections were then cut and stained using hematoxylin and eosin, prior to being used for routine histopathological examinations. All the tumors were diagnosed as IPMN. A representative tissue block was selected from each case and used for the immunohistochemical examinations.

Immunohistochemistry. The immunohistochemical staining of mesothelin was performed as previously described (8). Tissue sections (4- $\mu \mathrm{m}$ thick) were mounted on charged glass slides, deparaffinized and rehydrated through a graded ethanol series. For antigen retrieval, Dako Target Retrieval Solution (pH 9.0; catalogue number, S2368; Dako Denmark A/S, Glostrup, Denmark) was used, and the slides were boiled in a pressure cooker (Pascal Pressure Cooker; model, S2800; Dako North America, Inc., Carpinteria, CA, USA) at $125^{\circ} \mathrm{C}$ for $3 \mathrm{~min}$. The sections were treated with $0.3 \%$ hydrogen peroxidase for $5 \mathrm{~min}$ to quench endogenous peroxidase activity. Subsequently, the slides were incubated with a 1:50 dilution of a mouse monoclonal antibody for mesothelin (clone 5B2; Novocastra, Newcastle-Upon-Tyne, United Kingdom) at room temperature for $30 \mathrm{~min}$. The slides were then reacted with a dextran polymer reagent combined with horseradish peroxidase-conjugated secondary antibodies (Envision/HRP; Dako
North America, Inc.) for $30 \mathrm{~min}$ at room temperature. Specific antigen-antibody reactions were visualized using $0.2 \%$ diaminobenzine tetrahydrochloride and hydrogen peroxide. The slides were counterstained with hematoxylin for $10 \mathrm{~min}$ and then rinsed gently in reagent quality water.

Immunohistochemical evaluation. All assessments concentrated on the tumor-bearing regions of the specimens. Each slide was evaluated independently by three pathologists who were unaware of the clinical outcomes.

The immunostaining of mesothelin was evaluated in terms of the proportion of stained tumor cells and the staining intensity in each case. The proportion of immunostained mesothelin-positive cells was assessed as follows: $+1,1-10 \%$ of cells were stained; $+2,10-50 \%$ of cells were stained; and $+3,>50 \%$ of cells were stained. The mesothelin staining intensity was evaluated as weak (+1) or moderate to strong $(+2)$ and the localization of the staining was recorded as luminal membrane or cytoplasmic. The final mesothelin expression results for each case were then determined using the following scoring system, which was developed in a previous study of pancreatic cancer (8): mesothelin-positive was defined as a proportion score of $\geq+3$ and an intensity score of +2 , while mesothelin-negative was defined as a total score of $<+3$, except in cases involving a proportion score of +1 and an intensity score of +2 .

Furthermore, among the mesothelin-positive cases, the localization of mesothelin was evaluated as luminal membrane or cytoplasmic. Cases in which the entire circumference of the luminal membrane was clearly stained throughout the section were defined as luminal membrane-positive. Conversely, cases in which the luminal membrane was stained discontinuously or faintly, or cases in which no luminal membrane staining was observed, were defined as luminal membrane-negative. Cytoplasmic mesothelin expression was evaluated and cases in which cytoplasmic staining was clearly observed in the constituent cancer cells, including cytoplasmic granular staining, were defined as cytoplasm-positive (Fig. 1) (9).

Statistical analysis. The $\chi^{2}$-squared test or Fisher's exact test were used to determine the correlations between the mesothelin expression results and each clinicopathological parameter. All differences were considered significant at $\mathrm{P}<0.05$. All statistical analyses were performed using StatView 5.0 software (SAS Institute Inc., Cary, NC, USA).

\section{Results}

Mesothelin expression was detected in IPMN tissue, but not in the normal pancreatic tissue. All the benign pancreatic tissues were negative for mesothelin expression. Conversely, mesothelin expression was detected in adenoma and carcinoma cells. The majority of the adenoma cells that expressed mesothelin exhibited slight diffuse cytoplasmic staining (Fig. 2).

Recurrence of IPMN. The recurrence of IPMN was detected in six cases. The recurrence was located in the lymph nodes in two patients and in the peritoneum, liver and pleura in one patient each. 
Table I. Clinicopathological characteristics of the 37 IPMN patients.

\begin{tabular}{|c|c|c|c|c|}
\hline \multirow[b]{2}{*}{ Parameter } & \multirow[b]{2}{*}{ Total $(n=37)$} & \multicolumn{2}{|c|}{ Group } & \multirow[b]{2}{*}{ P-value } \\
\hline & & $\begin{array}{c}\text { Low-high } \\
\text { dysplasia }(n=26)\end{array}$ & $\begin{array}{c}\text { Invasive } \\
\text { carcinoma }(\mathrm{n}=11)\end{array}$ & \\
\hline Age, years $\pm \mathrm{SD}$ & $67.2 \pm 9.7$ & $65.7 \pm 9.7$ & $64.3 \pm 11.0$ & 0.71 \\
\hline \multicolumn{5}{|l|}{ Gender, $\mathrm{n}$} \\
\hline Male & 24 & 14 & 10 & 0.057 \\
\hline Female & 13 & 12 & 1 & \\
\hline \multicolumn{5}{|l|}{ Type of IPMN, n } \\
\hline Main duct or branch & 12 & 11 & 1 & 0.064 \\
\hline Combined & 25 & 15 & 10 & \\
\hline Mural nodules, n (\%) & $31(83.8)$ & $20(76.9)$ & $11(100.0)$ & 0.15 \\
\hline \multicolumn{5}{|l|}{ Recurrence, $\mathrm{n}$} \\
\hline Yes & 6 & 0 & 6 & 0.0002 \\
\hline No & 31 & 26 & 5 & \\
\hline
\end{tabular}

$\mathrm{SD}$, standard deviation; IPMN, intraductal papillary mucinous neoplasm.
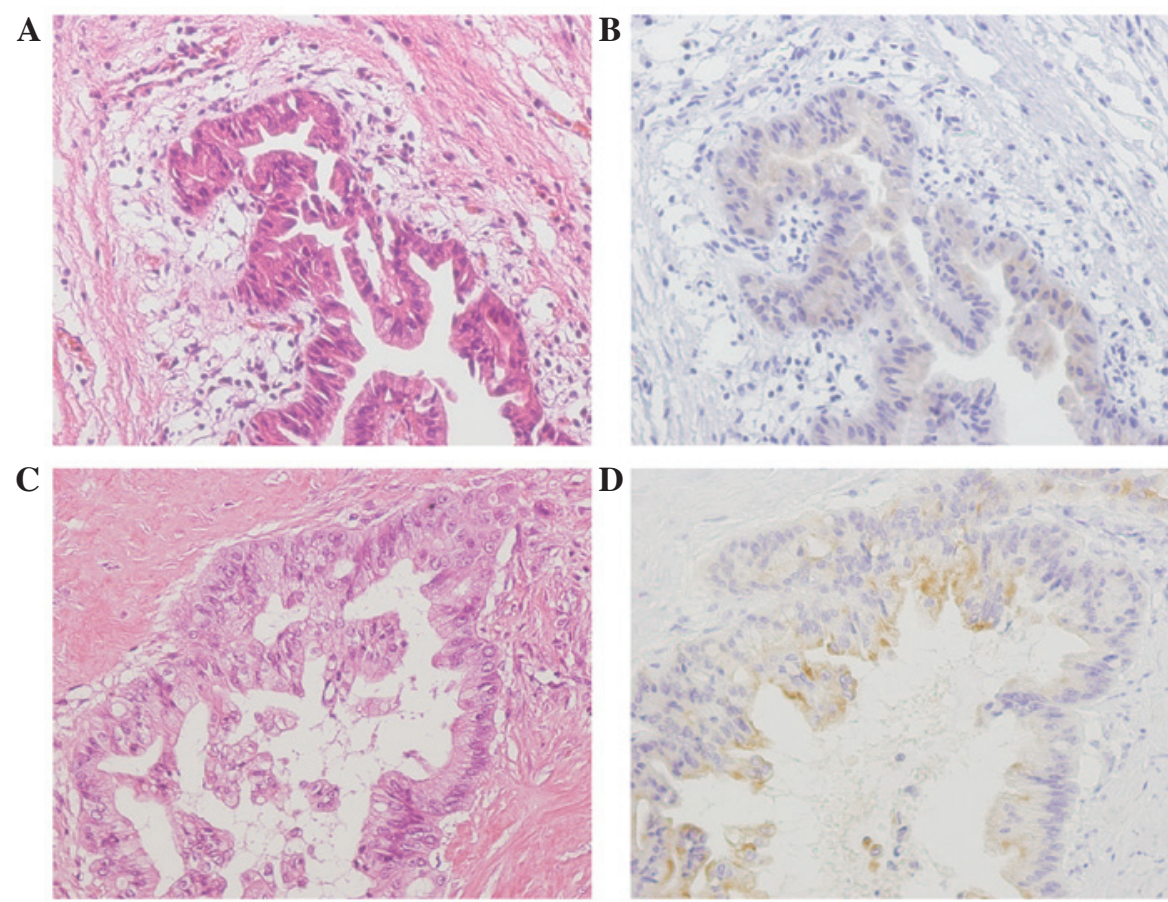

Figure 1. Representative samples of pancreatic ductal adenocarcinoma tissues that were awarded mesothelin expression scores of $0,+1$, and +2 . (A) Intraductal papillary mucinous adenoma tissue sample (stain, HE). (B) Mesothelin expression was faintly or barely detected in the tumor cell cytoplasm of (A) (mesothelin staining intensity of +1 ). No luminal membrane staining was detected in the adenoma cells. (C) Invasive intraductal papillary mucinous carcinoma (stain, HE). (D) Moderate to strong mesothelin expression was detected in the carcinoma cells (mesothelin staining intensity of +2 ). Granular cytoplasmic staining and staining of the entire cell membrane circumference were detected. This case was classified as luminal membrane-positive and cytoplasm-positive. Magnification, x200. HE, hematoxylin and eosin.

Mesothelin expression in IPMN. The findings of the present study regarding mesothelin expression are summarized in Table II. Among the 37 cases of IPMN, mesothelin expression was observed in $46.2 \%$ (12 out of 26) of the samples from L-H grade dysplasia and $81.8 \%$ (nine out of 11) of the samples from invasive carcinomas. Luminal membrane mesothelin expression was observed in $15.4 \%$ (four out of 26 ) of the L-H grade dysplasia samples and $54.5 \%$ (six out of 11) of the invasive carcinoma samples. Cytoplasmic mesothelin expression was observed in $38.5 \%$ (10 out of 26) of the $\mathrm{L}-\mathrm{H}$ grade dysplasias and $63.6 \%$ (seven out of 11) of the invasive carcinomas. The incidence of luminal membrane mesothelin expression was correlated with the histological classification of the tumor $(\mathrm{P}=0.022)$ and the recurrence rate $(\mathrm{P}=0.0030)$. There were no 
1586

EINAMA et al: LUMINAL MEMBRANE EXPRESSION OF MESOTHELIN IN IPMN

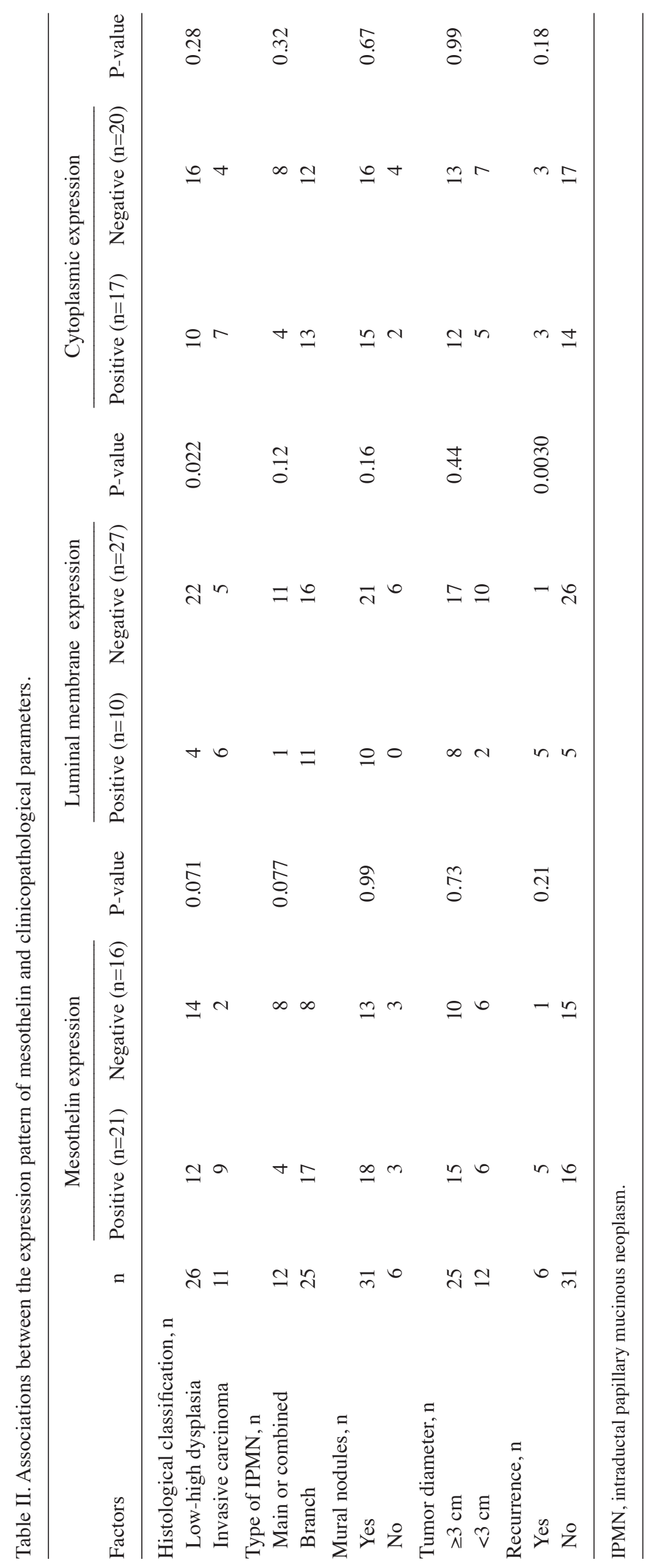


Table III. Association between histological classification and clinicopathological parameters.

\begin{tabular}{|c|c|c|c|c|}
\hline \multirow[b]{2}{*}{ Factors } & \multirow[b]{2}{*}{ Total $(n=37)$} & \multicolumn{2}{|c|}{ Group } & \multirow[b]{2}{*}{ P-value } \\
\hline & & $\begin{array}{c}\text { Low-high } \\
\text { dysplasia }(n=26)\end{array}$ & $\begin{array}{c}\text { Invasive } \\
\text { carcinoma }(\mathrm{n}=11)\end{array}$ & \\
\hline \multicolumn{5}{|l|}{ Type of IPMN, n } \\
\hline Main or combined & 12 & 11 & 1 & 0.064 \\
\hline Branch & 25 & 15 & 10 & \\
\hline \multicolumn{5}{|l|}{ Mural nodules, $\mathrm{n}$} \\
\hline Yes & 31 & 20 & 11 & 0.15 \\
\hline No & 6 & 6 & 0 & \\
\hline \multicolumn{5}{|l|}{ Tumor diameter, $\mathrm{n}$} \\
\hline$\geq 3 \mathrm{~cm}$ & 25 & 16 & 9 & 0.28 \\
\hline$<3 \mathrm{~cm}$ & 12 & 10 & 2 & \\
\hline \multicolumn{5}{|c|}{$\begin{array}{l}\text { Mesothelin membrane } \\
\text { expression, } \mathrm{n}\end{array}$} \\
\hline Yes & 10 & 4 & 6 & 0.022 \\
\hline No & 27 & 22 & 5 & \\
\hline
\end{tabular}

IPMN, intraductal papillary mucinous neoplasm.

Table IV. Mesothelin expression in patients with recurrent disease.

\begin{tabular}{|c|c|c|c|c|c|}
\hline \multirow[b]{2}{*}{ Case } & \multirow{2}{*}{$\begin{array}{l}\text { Age, } \\
\text { years }\end{array}$} & \multirow[b]{2}{*}{ Gender } & \multirow{2}{*}{$\begin{array}{l}\text { Mesothelin } \\
\text { expression }\end{array}$} & \multicolumn{2}{|c|}{ Mesothelin localization } \\
\hline & & & & Membrane & Cytoplasm \\
\hline 1 & 80 & M & + & + & + \\
\hline 2 & 80 & $\mathrm{M}$ & + & + & - \\
\hline 3 & 81 & M & + & + & + \\
\hline 4 & 55 & $\mathrm{M}$ & + & + & + \\
\hline 5 & 66 & M & + & + & + \\
\hline 6 & 72 & $\mathrm{~F}$ & - & - & - \\
\hline
\end{tabular}

M, male; F, female; +, positive; -, negative.

significant correlations between the histological classification and any of the other clinicopathological parameters (Table III).

The association between the mesothelin expression and recurrence of IPMN. Among the 37 IPMN patients, six suffered post-operative recurrence. In five of the cases with recurrent tumors, the tumors exhibited mesothelin expression, and all five tumors exhibited luminal membrane mesothelin expression (Table IV).

\section{Discussion}

In the present study, it was demonstrated that luminal membrane mesothelin expression in IPMN is associated with poor post-operative clinical outcomes. These results support the findings of previous studies investigating mesothelin expression in gastric cancer, extrahepatic bile duct cancer, and colorectal adenocarcinoma (9-11).
The possible mechanism responsible for the membranous localization of mesothelin may be based on the full-length human mesothelin gene encoding a 71-kDa precursor protein. This protein is proteolytically cleaved by furin-like proteases into an $\mathrm{N}$-terminal secreted form and a $\mathrm{C}$-terminal fragment, 40-kDa mesothelin, which is a GPI-linked glycoprotein $(1,13,14)$. The 5B2 anti-mesothelin antibody, which was employed in the immunohistochemical examination in the present study, is able to detect the $71-\mathrm{kDa}$ precursor protein and the $40-\mathrm{kDa} \mathrm{C}$-terminal fragment, but not the $30-\mathrm{kDa}$ $\mathrm{N}$-terminal fragment. Thus, based on the reported molecular processing mechanism of mesothelin and the specificity of the 5B2 antibody, the luminal membrane staining observed in the present study is likely to have indicated the presence of the 40-kDa membrane-bound form of mesothelin, while the cytoplasmic staining is likely to have indicated the presence of the 71-kDa precursor form of mesothelin. The present results are consistent with the results from previous studies, and 

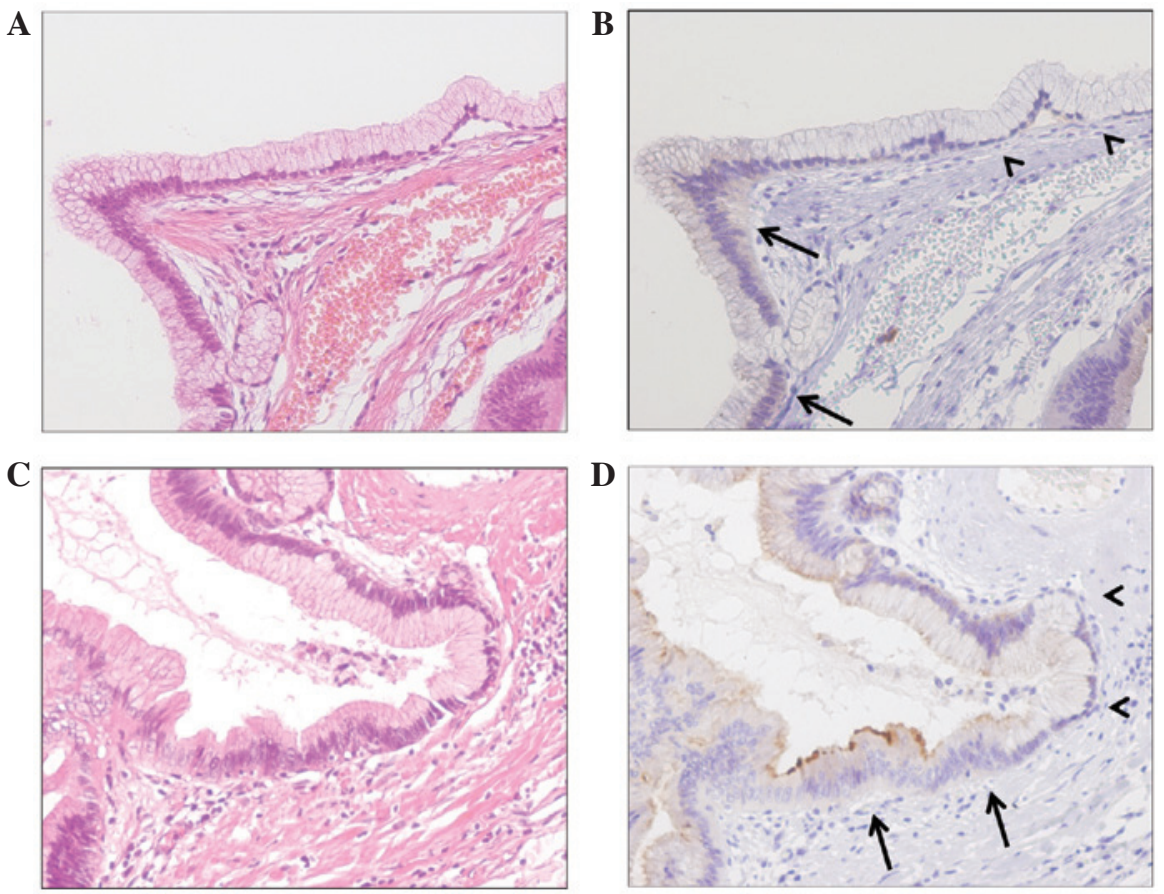

Figure 2. Mesothelin expression was detected in IPMN tissue, but not in the normal pancreatic tissue, and was limited to adenoma cells (arrows) Notably, the benign ductal epithelial cells of the intraductal papillary mucinous adenoma patients were negative for mesothelin expression (arrowheads). (A) Stain, hematoxylin and eosin (HE). (B) Tissue sample in (A) stained for mesothelin expression. (C) Stain, HE. (D) Tissue sample in (C) stained for mesothelin expression. Magnification, x200.

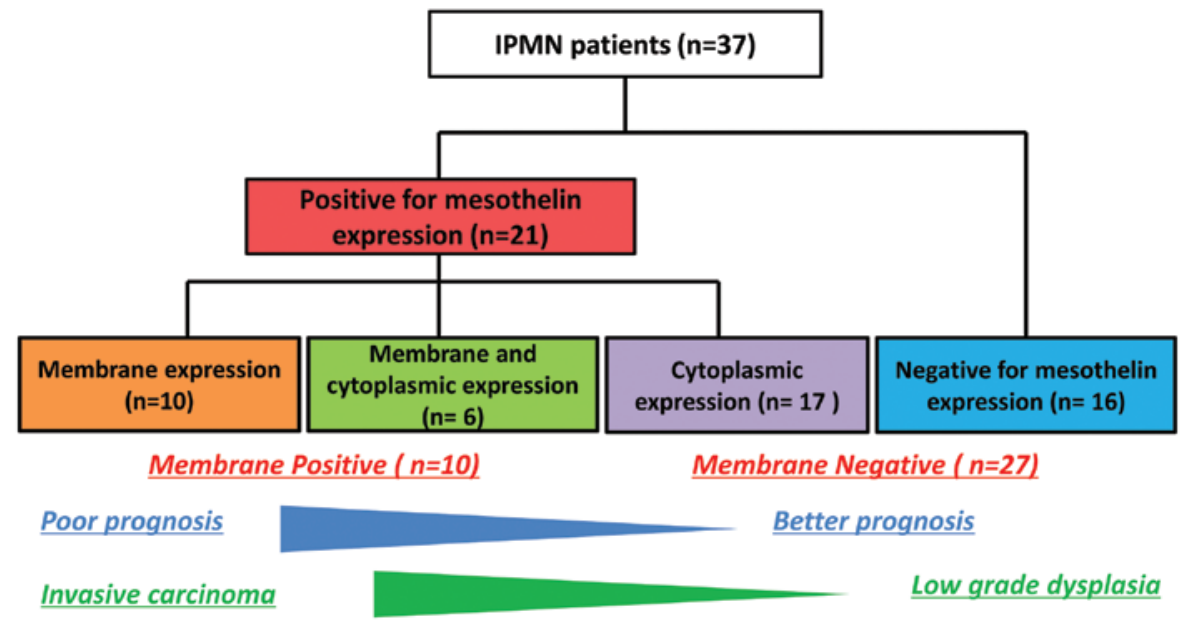

Figure 3. Flow chart of mesothelin expression in IPMN cells. IPMN, intraductal papillary mucinous neoplasm.

support the hypothesis that the 40-kDa membrane-bound form of mesothelin is the active form, which promotes aggressive cellular characteristics, including an increase in cell motility, invasive or migratory ability, and growth of metastatic tumors (15-17). In addition, Kawamata et al demonstrated that the biological function of $40-\mathrm{kDa}$ mesothelin is associated with lymphatic cancer cell invasion in vitro (10).

Pancreatic IPMNs exhibit a histological spectrum ranging between benign adenoma and invasive cancer (12). The cyst diameter, main pancreatic duct-type lesions and the presence of mural nodules are associated with histologically malignant grades of IMPN, and these criteria are widely used to exclude benign lesions from surgical intervention (18-20). At present, large numbers of benign lesions undergo surgical resection, which is suboptimal. As accurate pre-operative imaging-based assessments of malignancy are not currently possible, a method for identifying pre-invasive lesions and the establishment of a novel molecular-based management strategy is required. Appropriate criteria that can be used to identify IPMN containing rapidly invasive adenocarcinoma components are required. This would allow less aggressive lesions to simply be followed-up and avoid unnecessary surgery. Immunohistochemical evaluations of luminal membrane mesothelin expression in IPMN are considered to be of clinical benefit as they provide prognostic information (Fig. 3).

In conclusion, the present study demonstrated the prognostic significance of luminal membrane mesothelin expression in IPMN, although additional studies involving 
an increased number of luminal membrane-positive cases are required to confirm the present findings. Immunohistochemical examination of mesothelin expression in surgically resected tumor specimens is clinically useful for assessing the prognosis and for deciding on the necessity of additional treatment following surgical procedures in patients with IPMN.

\section{Acknowledgements}

The abstract was presented at the 2013 Gastrointestinal Cancers Symposium and published as abstract no. 179 in J Clin Oncol 31 (suppl 4, abstr 179), 2013. The authors would like to thank Mr. Moriya and Mr. Funayama for their technical assistance in immunohistochemistry.

\section{References}

1. Chang K and Pastan I: Molecular cloning of mesothelin, a differentiation antigen present on mesothelium, mesotheliomas, and ovarian cancers. Proc Natl Acad Sci USA 93: 136-140, 1996.

2. Chang K, Pastan I and Willingham MC: Isolation and characterization of a monoclonal antibody, $\mathrm{K} 1$, reactive with ovarian cancers and normal mesothelium. Int J Cancer 50: 373-381, 1992.

3. Argani P, Iacobuzio-Donahue C, Ryu B, et al: Mesothelin is overexpressed in the vast majority of ductal adenocarcinomas of the pancreas: identification of a new pancreatic cancer marker by serial analysis of gene expression (SAGE). Clin Cancer Res 7 : 3862-3868, 2001.

4. Hassan R, Kreitman RJ, Pastan I and Willingham MC: Localization of mesothelin in epithelial ovarian cancer. Appl Immunohistochem Mol Morphol 13: 243-247, 2005.

5. Ordóñez NG: Value of mesothelin immunostaining in the diagnosis of mesothelioma. Mod Pathol 16: 192-197, 2003.

6. Ordóñez NG: Application of mesothelin immunostaining in tumor diagnosis. Am J Surg Pathol 27: 1418-1428, 2003.

7. Li M, Bharadwaj U, Zhang R, et al: Mesothelin is a malignant factor and therapeutic vaccine target for pancreatic cancer. Mol Cancer Ther 7: 286-296, 2008.
8. Einama T, Kamachi H, Nishihara H, et al: Co-expression of mesothelin and CA125 correlates with unfavorable patient outcome in pancreatic ductal adenocarcinoma. Pancreas 40: 1276-1282, 2011

9. Einama T, Homma S, Kamachi H, et al: Luminal membrane expression of mesothelin is a prominent poor prognostic factor for gastric cancer. Br J Cancer May 29, 2012.

10. Kawamata F, Homma S, Kamachi H, et al: C-ERC/mesothelin provokes lymphatic invasion of colorectal adenocarcinoma. J Gastroenterol 49: 81-92, 2014.

11. Kawamata F, Kamachi H, Einama T, et al: Intracellular localization of mesothelin predicts patient prognosis of extrahepatic bile duct cancer. Int J Oncol 41: 2109-2118, 2012.

12. Bosman FT, Carneiro F, Hruban RH and Theise ND (eds): Tumors of the pancreas. In: WHO Classification of Tumours of the Digestive System. 4th edition. IARC press, Lyon, pp304-313, 2010.

13. Hassan R, Bera T and Pastan I: Mesothelin: a new target for immunotherapy. Clin Cancer Res 10: 3937-3942, 2004.

14. Cheng WF, Huang CY, Chang MC, et al: High mesothelin correlates with chemoresistance and poor survival in epithelial ovarian carcinoma. Br J Cancer 100: 1144-1153, 2009.

15. Bharadwaj U, Marin-Muller C, Li M, Chen C and Yao Q: Mesothelin overexpression promotes autocrine IL-6/sIL-6R trans-signaling to stimulate pancreatic cancer cell proliferation. Carcinogenesis 32: 1013-1024, 2011.

16. Bharadwaj U, Marin-Muller C, Li M, Chen C and Yao Q: Mesothelin confers pancreatic cancer cell resistance to TNF- $\alpha$-induced apoptosis through Akt/PI3K/NF- $\mathrm{BB}$ activation and IL-6/Mcl-1 overexpression. Mol Cancer 10: 106, 2011.

17. Inami K, Abe M, Takeda K, et al: Antitumor activity of anti-C-ERC/mesothelin monoclonal antibody in vivo. Cancer Sci 101: 969-974, 2010.

18. Sugiyama M, Izumisato Y, Abe N, et al: Predictive factors for malignancy in intraductal papillary-mucinous tumours of the pancreas. Br J Surg 90:1244-1249, 2003.

19. Schmidt CM, White PB, Waters JA, et al: Intraductal papillary mucinous neoplasms: predictors of malignant and invasive pathology. Ann Surg 246: 644-651, 2007.

20. Nara S, Onaya H, Hiraoka N, et al: Preoperative evaluation of invasive and noninvasive intraductal papillary-mucinous neoplasms of the pancreas: clinical, radiological, and pathological analysis of 123 cases. Pancreas 38:8-16, 2009. 